\title{
Apreciaciones sobre la quinta disciplina en la construcción de organizaciones educativas inteligentes
}

Appreciations about the fifth discipline in the construction of intelligent educational organizations

\section{Volumen 18, Número 3}

Setiembre-Diciembre

pp. 1-17

\author{
Johel Furguerle Rangel \\ José Pacheco Barrios \\ Gilberto Bastidas Pacheco
}

Revista indizada en REDALYC, $\underline{\text { SCIELO }}$

Revista distribuida en las bases de datos:

LATINDEX, DOAJ, REDIB, IRESIE, CLASE, DIALNET, SHERPA/ROMEO, QUALIS-CAPES, MIAR

Revista registrada en los directorios:

ULRICH'S, $\underline{\text { REDIE}}, \underline{\text { RINACE}}, \underline{\text { OEI }}$ MAESTROTECA, PREAL, $\underline{\text { CLACSO }}$ 


\title{
Apreciaciones sobre la quinta disciplina en la construcción de organizaciones educativas inteligentes
}

\author{
Appreciations about the fifth discipline in the construction of intelligent educational
} organizations

\author{
Johel Furguerle Range ${ }^{1}$ \\ José Pacheco Barrios ${ }^{2}$ \\ Gilberto Bastidas Pacheco ${ }^{3}$
}

\begin{abstract}
Resumen: En las instituciones educativas, los aspectos organizativos han sido relegados porque la política educativa se ha esmerado en producir nuevos proyectos curriculares atrapados en marcos organizativos arcaicos, con la consiguiente imposibilidad para el logro de los objetivos institucionales. Bajo esta consideración, en este artículo, se evaluaron las actitudes que las personas docentes poseen hacia las organizaciones inteligentes basadas en la quinta disciplina de Serge, la cual expresa la construcción del pensamiento sistémico. Se utilizó una muestra de 40 docentes del Liceo Bolivariano "Dr. Burelli Rivas", ubicado en el municipio Valera, estado Trujillo, Venezuela. La investigación fue de campo, transversal, de tipo descriptiva. Se recurrió a la encuesta para recoger datos, y como instrumento se aplicaron dos cuestionarios autoadministrados, escala tipo Likert, previa obtención de consentimiento informado. La apreciación del cuerpo docente fue alta en cuanto a dominio personal, modelos mentales y pensamiento sistémico, y fue media, respecto a todos los aspectos que definen las organizaciones inteligentes. Se concluye que el grupo docente encuestado tiene actitud positiva por el pensamiento sistémico de la quinta disciplina, por ser la integradora de las demás doctrinas o postulados. Sin embargo, las personas docentes creen medianamente importante la práctica de la visión compartida; es decir, la creencia genuina de un futuro común para el logro de los objetivos de las instituciones educativas.
\end{abstract}

Palabras clave: quinta disciplina, organizaciones inteligentes, instituciones educativas, conocimiento.

\begin{abstract}
In the educational institutions, the organizational aspects have been relegated because the educational policy has been careful to produce new curricular projects trapped in archaic organizational frameworks, with the consequent impossibility for the achievement of the institutional objectives. Under this consideration, in this article, the attitudes that teachers have towards intelligent organizations based on Serge's fifth discipline, which expresses the construction of systems thinking, were evaluated. A sample of 40 teachers from the high school Bolivariano "Dr. Burelli Rivas "located in the municipality of Valera, Trujillo state, Venezuela. The investigation was field, transversal, descriptive type. The survey was used to collect data and as an instrument two self-administered Likert-type questionnaires were applied, after obtaining informed consent. Teacher appreciation was high in terms of personal mastery, mental models, and systemic thinking, and was media, with respect to all aspects that define intelligent organizations. It is concluded that the teachers surveyed have a positive attitude towards the systemic thinking of the fifth discipline, since it is the integrator of the other doctrines or postulates. However, teachers believe that the practice of shared vision is fairly important, that is, the genuine belief of a common future, for the achievement of the objectives of educational institutions.
\end{abstract}

Keywords: fifth discipline, intelligent organizations, educational institutions, knowledge.

\footnotetext{
1 Universidad Nacional Experimental "Rafael María Baralt", Venezuela. Doctor en Gerencia Educativa. Dirección electrónica: ihoelfurguerle@hotmail.com

2 Universidad Nacional Experimental "Rafael María Baralt", Venezuela. Magíster Scientarium en Administración de la Educación Básica. Dirección electrónica: Email: pachecobarriosj29@hotmail.com
}

3 Universidad de Carabobo, Departamento de Salud Pública y Centro de Investigaciones Medicas y Biotecnológicas, Facultad de Ciencias de la Salud, Venezuela. Doctor en Parasitología. Dirección electrónica: bastidasprotozoo@hotmail.com

Artículo recibido: 8 de diciembre, 2017 Enviado a corrección: 11 de junio, 2018 Aprobado: 27 de agosto, 2018 


\section{Introducción}

El origen de las organizaciones se remonta al inicio de la humanidad por la misma necesidad del hombre de agruparse para protegerse y satisfacer sus necesidades básicas de procurarse alimento, refugio y conocimiento tanto para sí mismo, como para los suyos, por eso, se nace, vive, educa y trabaja en las organizaciones, de allí que se privilegie el estudio de las mismas, ya que, al ser un producto del hombre carece de perfección, sin embargo, se persigue la misma, de allí el desarrollo de teorías gerenciales y administrativas (clásicas y las que versan sobre relaciones humanas) aportadas por diversos investigadores desde comienzos del siglo XX (Guizar, 2004; Bastidas y Pacheco, 2011; Furguerle, Pacheco, Hernández y Bastidas, 2016).

Asimismo, con el crecimiento de la población, la expansión global de la tecnología y el requerimiento de bienes y servicios cada vez más específicos, las organizaciones han debido diversificarse, desde las más simples hasta las más complejas, con sus características particulares, pero todas orientadas al concepto de la eficiencia. En este sentido se señala que el conocimiento y la gestión son verdaderamente importantes como alternativa para lograr la eficiencia de una organización ante los retos que plantee el medio donde se encuentra inserta, porque corresponde al conocimiento, mejor dicho a la adquisición del mismo, el mantenimiento de la vigencia en el tiempo de la organización, (Senge, 1993, 2005; León et al., 2003; Casell y Perea, 2009).

Por este motivo, en el desarrollo organizacional se privilegia el aprendizaje como la herramienta eficaz para transformar los esquemas mentales de la organización y conducirla hacia el logro de sus metas. Si cada organización en conjunto con sus miembros devela su propia realidad, entonces el aprendizaje no sólo en el plano individual, sino grupal, contribuirá eficaz y responsablemente a la comprensión de los escenarios, en pleno acuerdo con la relación, por demás estrecha, que existe entre conocimiento, aprendizaje y eficiencia tradicionalmente asociada a organizaciones con fines de lucro (organización inteligentes), pero sin duda también válida para las educativas (Aguerrondo, 1992, 1996; León et al., 2003).

El campo educativo, no escapa a la necesidad de beneficiarse de las teorías organizativas que apuntan hacia el conocimiento para regular su funcionamiento, porque empresas y escuelas tienen más puntos en común de lo que se cree, uno de ellos es el compromiso con los resultados, en el caso del sector educativo como proveedor de un servicio caracterizado por formar seres humanos en aspectos técnicos y con base en los 
valores éticos y morales, por tanto, el sector educativo debe estar naturalmente inclinado hacia la búsqueda permanente de la calidad y la excelencia; especialmente cuando muchos sistemas educativos, con ligeros a graves fracasos, únicamente innovan hacia el aspecto académico (fundamentalmente dirigido a mejorar la actuación del docente en el aula) dejando de lado la organización, y especialmente lo que piensan los actores educativos sobre las teorías gerenciales, entre ellas la del conocimiento, objeto del presente estudio (Aguerrondo, 1992; León et al., 2003).

En la actualidad, según Senge (1990), en las organizaciones inteligentes convergen cinco nuevos criterios (pensamiento sistémico, dominio personal, modelos mentales, construcción de una visión compartida y aprendizaje en equipo), que por separado cada cual proporciona un fascículo crucial para la construcción de organizaciones con auténtica capacidad de aprendizaje, pero que integradas en un cuerpo teórico coherente como la quinta disciplina (como pensamiento sistémico) y en el entendido que el todo supera la suma de las partes, asegura el máximo aprovechamiento del entusiasmo y la capacidad de aprendizaje del docente, como ser sujeto y objeto de la intervención, en cualquier nivel de la organización educativa.

Las ideas presentadas hasta aquí fundamentan el objeto de esta investigación, específicamente, la determinación de las apreciaciones que los docentes poseen sobre la quinta disciplina como pensamiento sistémico para la organización inteligente, bajo la premisa de que las instituciones educativas son aquellas donde la gente acrecenta continuamente su actitud para obtener los resultados pedagógicos deseados, donde se producen nuevos y expansivos patrones de pensamiento, donde se deja en libertad la aspiración colectiva y se impulsa en el grupo la necesidad de aprender a aprender (Gil, 2006). Por tanto, es vital develar la actitud del docente sobre la quinta disciplina en instituciones educativa donde antes no se ha investigado hacia la formulación de una visión cónsona con la realidad organizacional.

\section{El problema}

Los aspectos organizativos en el campo educativo han sido tradicionalmente relegados, especialmente porque las políticas educativas del Estado se han esmerado en producir nuevos proyectos, pero con novedades curriculares atrapadas en marcos organizativos arcaicos, con el consiguiente desequilibrio en el logro de los objetivos que la institución se propone, bajo esta consideración se señala que el fracaso en los intentos por 
mejorar la educación en América Latina se debe a la excesiva preocupación por lo académico cuyo centro es el aula y, al descuido de la estructura y funcionamiento escolar, es decir, se considera al aula como la unidad de transformación de la educación y no a la institución (Aguerrondo, 1992, 1996).

Por consiguiente, las instituciones educativas, llamadas a cumplir con los parámetros de desarrollo social establecidos en el marco educativo del país, requieren convertirse, se cree firmemente, en organizaciones inteligentes, con base en la quinta disciplina, como herramienta gerencial que conlleve a su transformación tanto interna como externa, expresada en términos de clarificación de su misión, cambio de visión con perspectiva de futuro, fomento del aprendizaje continuo en todos sus miembros, así como de una real apertura hacia el entorno. Para ello, precisa la práctica a nivel individual del dominio personal y los modelos mentales, y a nivel del grupo la visión compartida, el aprendizaje en equipo y el pensamiento sistémico (Senge, 1993, 2005; Gómez, 2012).

De modo que, cada quien desde su posición expanda sus pensamientos, su creatividad en la búsqueda de mejorar y actualizar sus conocimientos mediante la interacción con otros, no sólo desde el punto de vista de su desarrollo personal, sino para el crecimiento de su equipo de trabajo. Entonces, lo que distinguirá fundamentalmente las organizaciones inteligentes de las tradicionales y autoritarias "organizaciones de control" es el dominio de la capacidad de aprender, la resolución de problemas considerando sus efectos futuros, con el fin de evitar reacciones colaterales o incluso la repetición de los mismos a largo plazo (Llano, 1998; León et al., 2003; Carballo, Masís, Sárraga y Solís, 2008; Gil, 2006; Covey, 2009).

Esta apuesta por la práctica de la quinta disciplina responde al alto número de instituciones educativas que mantienen esquemas organizativos de tipo jerárquico, con directores en un rol de controlador de todo el accionar institucional, que no delega funciones y menos aún cuotas de poder a su personal para la toma de decisiones y resolución de problemas, con la consecuente pérdida del compromiso personal con la institución educativa donde se labora, que potencia el ausentismo, la impuntualidad, genera fallas en la comunicación interpersonal, dificulta la coordinación de actividades y por tanto, resulta en escasa participación en actividades conjuntas. Igualmente se ve justificada la investigación sobre el modelo gerencial sistémico de la quinta disciplina, específicamente de las actitudes o apreciaciones que sobre el mismo poseen los docentes, por el desconocimiento o escaso conocimiento que se tiene sobre este tipo de herramienta organizacional (Senge, 2005). 
Especialmente porque se ha demostrado la utilidad en estructuras organizativas educativas, de los postulados teóricos de la quinta disciplina, específicamente como coadyuvante en el afrontamiento de los necesarios cambios que deben producirse en los plantes educativos ante el entorno, siempre cambiante, por supuesto en plena consideración de la necesaria innovación en la labor de formar jóvenes aptos para la vida productiva en sociedad, en que los tradicionales modelos gerenciales parecen no brindar los resultados deseados y donde tiene cabida la implementación de nuevas o poco probadas técnicas gerenciales, claro está previa determinación del conocimiento y actitud que los involucrados tienen sobre las mismas, como requisito precedente para medir su efectividad en la loable labor de formar al ciudadano (Bolívar, 2000; Senge, 2005).

En este estudio se mide las actitudes o apreciaciones, que tienen los docentes, sobre la conversión de las instituciones educativas en organizaciones inteligentes, específicamente en aquellas, como los liceos bolivarianos venezolanos (donde se forman los adolescentes), que deben elaborar proyectos de aprendizaje con la participación de todos como estrategia para lograr la enseñanza efectiva que incluye el abordaje de un particular problema académico o social de interés o relevancia para el colectivo, porque en las organizaciones inteligentes todos los miembros cultivan permanentemente la necesidad de aprender, principalmente de aprender en conjunto (Ministerio de Educación y Deportes, 2004; Ministerio del Poder Popular para la Educación, 2007).

Por tanto, se precisa el estudio de las apreciaciones que sobre las herramientas gerenciales posee el docente, objeto de este estudio, particularmente de la quinta disciplina y de las organizaciones inteligentes, como paso previo y necesario en la construcción de un verdadero proceso de integración de saberes, de la inter y transdisciplinariedad, del compartir con los demás, de la curiosidad, de la asimilación del cambio y fundamentalmente del diálogo, todos elementos definitorios de la activación del aprendizaje de la organización y requisitos indispensables en la construcción de instituciones educativas exitosas (Llano, 1998; Carballo et al., 2008).

\section{Metodología}

\subsection{Tipo y diseño de la investigación}

La investigación fue de campo, cualitativa, transversal, de tipo ontológico y descriptiva con base en la observación directa y objetiva de la realidad y el registro de los hechos tal y como se presentan en su entorno natural, a través, de la medición de las variables que 
componen la quinta disciplina como pensamiento sistémico de la organización inteligente, para determinar la percepción que sobre la misma poseen los docentes del Liceo Bolivariano "Dr. Burelli Rivas" ubicado en el municipio Valera, estado Trujillo, Venezuela; un modelo típico de liceo regido por proyectos educativos en la formación de adolescentes, como individuos que inician la compresión e integración, ya consciente o madura en la sociedad que los acogió como niños.

\subsection{Población y muestra}

En este estudio se incluyo a todos los docentes, 40 en total, del Liceo Bolivariano "Dr. Burelli Rivas", ubicado en la parroquia Mendoza del municipio Valera, los mismos fueron seleccionados por tener su carga horaria concentrada en la institución y por poseer conocimientos formales en materia de diseño curricular.

\subsection{Técnica de recolección de datos}

Se utilizó el cuestionario, como técnica con el fin de obtener las apreciaciones que los docentes tienen sobre la quinta disciplina (sus componentes son: dominio personal, modelos mentales, construcción de una visión compartida, aprendizaje en equipo y pensamiento sistémico) y la organización inteligente (sus componentes son: uso del conocimiento, enfoque, resolución de problemas, sistema, actitud ante el cambio, capacitación, uso de la comunicación e interacción con el entorno), en esta era del conocimiento donde particularmente las instituciones educativas tienen la necesidad de ampliar y potenciar el talento que permita potenciar las ventajas competitivas en cuanto a formación que exige, el constate cambio, de este mundo globalizado a las organizaciones educativas. Previamente a la obtención de la información se proporcionó a los docentes suficiente información sobre los postulados de la quinta disciplina y la organización inteligente, para evitar sesgos por desconocimiento del tema.

La recolección de datos mediante la encuesta ameritó la aplicación de dos cuestionarios auto administrados con alternativas de respuestas cerradas (Tabla 1 y Tabla 2), uno para medir las apreciaciones que sobre la variable quinta disciplina tienen los docentes de la institución educativa seleccionada, compuesto por 20 preguntas, y otro para investigar la variable organización inteligente; ambos cuestionarios son tipo Likert con una escala de 5 puntos que mide el nivel de frecuencia con las categorías siempre, casi siempre, algunas veces, casi nunca y nunca. Los instrumentos fueron previamente validados, a través, 
de juicios de expertos y la confiabilidad de los datos se aseguró con pruebas pilotos (previa obtención de consentimiento informado) para el cálculo del coeficiente Alfa de Cronbach, que para la variable quinta disciplina alcanzo un valor $\alpha=0,83$, y para la variable organización inteligente fue de $\alpha=0,92$.

Tabla 1

Ítems empleados para determinar las apreciaciones sobre la quinta disciplina de docentes del Liceo Bolivariano "Dr. Burelli Rivas”. Municipio Valera, Trujillo, Venezuela. 2016.

\begin{tabular}{|c|c|c|}
\hline Indicadores & Ítems & $\mathbf{N}^{\circ}$ \\
\hline \multirow{2}{*}{$\begin{array}{l}\text { Dominio } \\
\text { personal }\end{array}$} & Tolerancia ante críticas realizadas por otros miembros de la institución. & 1 \\
\hline & $\begin{array}{l}\text { Participación en actividades de crecimiento personal. } \\
\text { Manejo de emociones de manera adecuada ante la discrepancia de opinión } \\
\text { con otros miembros de la institución. } \\
\text { Adopción de la práctica de una continúa superación personal. }\end{array}$ & $\begin{array}{l}3 \\
4\end{array}$ \\
\hline $\begin{array}{l}\text { Modelos } \\
\text { mentales }\end{array}$ & $\begin{array}{l}\text { Interacción igualitaria con todos los miembros de la institución. } \\
\text { Trabajo enmarcado dentro de la planificación de la institución. } \\
\text { Visualización de problemas de organización en la institución. } \\
\text { Incentivado a actualizarse en cuanto al uso de las tecnologías de la } \\
\text { información y la comunicación. }\end{array}$ & $\begin{array}{l}5 \\
6 \\
7\end{array}$ \\
\hline $\begin{array}{l}\text { Construcción } \\
\text { de una visión } \\
\text { compartida }\end{array}$ & $\begin{array}{l}\text { Participación en la creación de la visión institucional. } \\
\text { Misión de la institución compartida. } \\
\text { El liderazgo ejercido por el directivo, conduce al logro de los objetivos } \\
\text { institucionales. } \\
\text { El trabajo desarrollado por el docente le permite sentirse parte fundamental } \\
\text { del desarrollo de la institución. }\end{array}$ & $\begin{array}{l}9 \\
10\end{array}$ \\
\hline $\begin{array}{l}\text { Aprendizaje } \\
\text { en equipo }\end{array}$ & $\begin{array}{l}\text { Apertura para aprender de otros. } \\
\text { Planificación de la labor en conjunto con otros integrantes de la institución. } \\
\text { Recepción oportuna de la información acerca de la labor de los demás } \\
\text { integrantes de la institución. } \\
\text { Diálogo abierto con los demás miembros de la institución al desarrollar } \\
\text { actividades en conjunto. }\end{array}$ & $\begin{array}{l}13 \\
14 \\
15\end{array}$ \\
\hline $\begin{array}{l}\text { Pensamiento } \\
\text { sistémico }\end{array}$ & $\begin{array}{l}\text { Reconocimiento de corresponsabilidad ante los problemas presentados por } \\
\text { la institución. } \\
\text { Practica de la visión sistémica en la planificación de su labor. } \\
\text { Involucramiento del docente en las actividades planificadas con la } \\
\text { participación de todos los entes del sistema educativo. } \\
\text { Labor desarrollada en correspondencia con las demandas de la sociedad } \\
\text { actual. }\end{array}$ & $\begin{array}{l}17 \\
18 \\
19\end{array}$ \\
\hline
\end{tabular}

Fuente: Elaborado por los investigadores, 2016. 
Tabla 2

Ítems empleados para determinar las apreciaciones sobre la organización inteligente de docentes del Liceo Bolivariano "Dr. Burelli Rivas”. Municipio Valera, Trujillo, Venezuela. 2016.

\begin{tabular}{|c|c|c|}
\hline \multirow{3}{*}{$\begin{array}{c}\text { Indicadores } \\
\text { Uso del } \\
\text { conocimiento }\end{array}$} & İtems & $\mathbf{N}^{\circ}$ \\
\hline & Actualización del conocimiento en sus integrantes. & 1 \\
\hline & $\begin{array}{l}\text { Promoción del conocimiento de la metodología de trabajo del Liceo } \\
\text { Bolivariano. } \\
\text { Incentivo a la práctica del conocimiento colaborativo. }\end{array}$ & \\
\hline Enfoque & $\begin{array}{l}\text { Permiso a miembros para variar las estrategias de trabajo. } \\
\text { Estimulo a los integrantes de la institución, a cumplir con su labor más allá } \\
\text { del horario establecido. } \\
\text { Se propicia la capacitación de sus miembros para asumir funciones de } \\
\text { liderazgo al planificar actividades colectivas. }\end{array}$ & 4 \\
\hline $\begin{array}{l}\text { Resolución de } \\
\text { problemas }\end{array}$ & $\begin{array}{l}\text { Todos contribuyen con la solución de problemas de la institución. } \\
\text { Se fomenta el trabajo en conjunto hacia la solución de problemas a largo } \\
\text { plazo. } \\
\text { Fomento de reuniones de trabajo productivas a fin de encontrar soluciones a } \\
\text { las necesidades. }\end{array}$ & 7 \\
\hline Sistema & $\begin{array}{l}\text { Visualización de la institución como un sistema del cual cada integrante } \\
\text { forma parte importante. } \\
\text { Existencia de una relación entre el trabajo de todos los miembros de la } \\
\text { institución. } \\
\text { Desarrollo del trabajo interdisciplinario como estrategia para la práctica de la } \\
\text { visión sistémica. }\end{array}$ & 11 \\
\hline $\begin{array}{l}\text { Actitud ante el } \\
\text { cambio }\end{array}$ & $\begin{array}{l}\text { Se asume el cambio como parte del desarrollo evolutivo de la institución. } \\
\text { Acatamiento de los cambios emanados del sistema educativo. } \\
\text { Se estimula a todos los integrantes a adoptar el cambio como herramienta } \\
\text { para el mejoramiento de su desempeño. }\end{array}$ & $\begin{array}{l}13 \\
14\end{array}$ \\
\hline Cape & $\begin{array}{l}\text { Estimulo para que todos los integrantes de la institución se capaciten } \\
\text { permanentemente. } \\
\text { Se fomenta el compromiso de todos los integrantes con el desarrollo óptimo } \\
\text { de su labor. } \\
\text { Valoración de la iniciativa particular de sus integrantes hacia el crecimiento } \\
\text { profesional, a través de la prosecución de estudios. }\end{array}$ & 16 \\
\hline $\begin{array}{l}\text { Usc } \\
\text { comu }\end{array}$ & $\begin{array}{l}\text { Se promueve la comunicación asertiva entre los miembros de la institución. } \\
\text { Se muestra una adecuada comunicación entre el personal directivo y los } \\
\text { demás integrantes de la institución. } \\
\text { El flujo de información en cuanto a las actividades planificadas llega } \\
\text { convenientemente a todos sus integrantes. }\end{array}$ & 20 \\
\hline $\begin{array}{c}\text { Interacción } \\
\text { con el entorno }\end{array}$ & $\begin{array}{l}\text { Trabajo vinculado con la comunidad mediante el desarrollo del Proyecto } \\
\text { Educativo Integral Comunitario. } \\
\text { Visualización del sentido de pertenencia de parte de la comunidad hacia el } \\
\text { liceo. } \\
\text { Participación en actividades organizadas por la comunidad, con el fin de } \\
\text { buscar solución a problemas comunitario. }\end{array}$ & 22 \\
\hline
\end{tabular}

Fuente: Elaborado por los investigadores 2016.

Para el tratamiento de la información, se diseñó una escala de valores desde uno (01) hasta cinco (05), distribuidos con igual cantidad de elementos dentro de cada intervalo. Los 
mismos se determinaron con la ecuación: máximo valor-mínimo valor/número máximo de la escala. El valor obtenido se suma a cada numero entero partiendo del 1 de esta manera se obtienen los intervalos para cada una de los ítems, a los cuales, se les asignó un criterio de valoración cualitativa, tal como se evidencia en el Tabla 3.

Tabla 3

Rangos y criterios para la valoración de las variables quinta disciplina y organización inteligente de docentes del Liceo Bolivariano "Dr. Burelli Rivas". Municipio Valera, Trujillo, Venezuela. 2016.

\begin{tabular}{cc}
\hline Rango & Criterio \\
\hline $1<1,9$ & Muy bajo \\
$1,9<2,8$ & Bajo \\
$2,8<3,7$ & Medio \\
$3,7<4,6$ & Alto \\
$4,6-5$ & Muy alto \\
\hline
\end{tabular}

Fuente: Elaborado por los investigadores, 2016.

Los datos obtenidos fueron vaciados en una hoja de cálculo en Excel® creada para tal fin. A la información en frecuencias absolutas y relativas (proporciones) se le realizó estadística descriptiva, principalmente las de tendencia central (media).

\section{Resultados}

La apreciación que los docentes tienen respecto al dominio personal de la quinta disciplina como herramienta gerencial, es alta, con un promedio de respuesta a la sumatoria de todas las alternativas de 4,0, es por ello que puede señalarse que los docentes consideran la práctica de la tolerancia hacia las críticas que le realizan los otros miembros de la institución, el constante crecimiento personal (alta disposición hacia la superación personal, es decir, a la práctica de la auto-superación) y el control emocional ante las discrepancias como útiles para alcanzar la transformación de su institución educativa hacia la deseada organización inteligente (Tabla 4).

Los modelos mentales, el otro componente con carácter individual de la quinta disciplina (al que se suma el dominio personal), tiene alta aceptación por los docentes estudiados, con un promedio de 3,7, fundamentalmente sustentado este resultado en la mayor relevancia que los encuestados otorgan a la capacidad del individuo para visualizar los problemas de organización en la institución y al trabajo dentro de la planificación del liceo; pues los docentes, dentro del componente modelo mental, otorgan mediana importancia a la igual interacción con todos los miembros de la institución y a la actualización en el uso de tecnologías de la información y comunicación (Tabla 4). 
Al indagarse sobre los componentes colectivos o grupales de la quinta disciplina, se determinó que los docentes ubican en la categoría cualitativa alta al pensamiento sistémico (promedio de 3,7 para este indicador), como útil para el fortalecimiento y desarrollo de la institución educativa de la cual se forma parte, y como medio la visión compartida (en relación al establecimiento de metas futuras) y el aprendizaje en equipo, aunque creen, por un lado (como parte de la visión compartida), al darles altos promedios, que compartir la misión de la institución (3,9 de promedio para el ítem) y que el trabajo realizado por el docente le permitirá sentirse parte fundamental del desarrollo de la institución (3,8 de promedio para el ítem) y por otro, en este caso con el aprendizaje en equipo, catalogan de alta la corresponsabilidad ante los problemas (3,9 de promedio para el ítem) y la labor desarrollada en correspondencia con las demandas de la sociedad (3,8 de promedio para el ítem) (Tabla 4).

Tabla 4

Puntuación promedio por cada ítem e indicador y según apreciaciones sobre los componentes de la quinta disciplina de los docentes del Liceo Bolivariano "Dr. Burelli Rivas". Municipio Valera, Trujillo, Venezuela. 2016.

\begin{tabular}{|c|c|c|c|c|}
\hline Indicador & Ítem & $\begin{array}{c}\text { Puntuación } \\
\text { promedio } \\
\text { del Ítem }\end{array}$ & $\begin{array}{l}\text { Puntuación } \\
\text { promedio del } \\
\text { Indicador }\end{array}$ & $\begin{array}{c}\text { Apreciación } \\
\text { cualitativa }\end{array}$ \\
\hline & 1 & 4 & & \\
\hline \multirow{4}{*}{ Dominio personal } & 2 & 4 & 4,0 & Alto \\
\hline & 3 & 4,1 & & \\
\hline & 4 & 4,1 & & \\
\hline & 5 & 3,6 & & \\
\hline \multirow[t]{4}{*}{ Modelos mentales } & 6 & 4,2 & 3,7 & Alto \\
\hline & 7 & 3,7 & & \\
\hline & 8 & 3,4 & & \\
\hline & 9 & 3,4 & & \\
\hline \multirow[t]{4}{*}{ Visión compartida } & 10 & 4,1 & 3,6 & Medio \\
\hline & 11 & 3,3 & & \\
\hline & 12 & 3,8 & & \\
\hline & 13 & 3,9 & & \\
\hline \multirow{5}{*}{$\begin{array}{l}\text { Aprendizaje } \\
\text { equipo }\end{array}$} & & & & \\
\hline & 14 & 3,6 & 3,6 & Medio \\
\hline & 15 & 3,2 & & \\
\hline & 16 & 3,8 & & \\
\hline & 17 & 3,9 & & \\
\hline \multirow{4}{*}{$\begin{array}{l}\text { Pensamiento } \\
\text { sistémico }\end{array}$} & & & & \\
\hline & 18 & 3,7 & 3,7 & Alto \\
\hline & 19 & 3,3 & & \\
\hline & 20 & 3,9 & & \\
\hline
\end{tabular}

Fuente: Elaboración propia, 2016. 
Los docentes del Liceo Bolivariano "Dr. Burelli Rivas", en todos los indicadores que miden las apreciaciones de estos sobre la organización inteligente, expresan como medio su influencia en el éxito que la aplicación de las mismas tiene en la formación de ciudadanos, sin embargo, a 6 interrogantes los docentes adjudicaron alta calificación cualitativa a saber: la actualización del conocimiento en sus integrantes (3,8 de promedio para el ítem [indicador uso del conocimiento]), permiso a miembros para variar las estrategias de trabajo (3,7 de promedio para el ítem [indicador enfoque]), se asume el cambio como parte del desarrollo evolutivo de la institución (3,7 de promedio para el ítem [indicador actitud ante el cambio]), fomento del compromiso de todos los integrantes con el desarrollo óptimo de su labor (3,9 de promedio para el ítem [indicador capacitación]) y la adecuada comunicación entre el personal directivo y los demás integrantes de la institución (3,7 de promedio para el ítem [indicador uso de la comunicación]) (Tabla 5).

Tabla 5

Puntuación promedio por cada ítem e indicador y según apreciaciones sobre los componentes de la organización inteligente de los docentes del Liceo Bolivariano "Dr. Burelli Rivas". Municipio Valera, Trujillo, Venezuela. 2016.

\begin{tabular}{|c|c|c|c|c|}
\hline Indicador & Ítem & $\begin{array}{c}\text { Puntuación } \\
\text { promedio } \\
\text { del Ítem }\end{array}$ & $\begin{array}{c}\text { Puntuación } \\
\text { promedio } \\
\text { Indicador }\end{array}$ & $\begin{array}{l}\text { Apreciación } \\
\text { cualitativa }\end{array}$ \\
\hline & 1 & 3,8 & \multirow{4}{*}{3,6} & \multirow{4}{*}{ Medio } \\
\hline \multirow{3}{*}{ Uso del conocimiento } & 2 & 3,5 & & \\
\hline & 3 & 3,6 & & \\
\hline & 4 & 3,7 & & \\
\hline \multirow{3}{*}{ Enfoque } & 5 & 3,1 & \multirow[t]{3}{*}{3,3} & \multirow{3}{*}{ Medio } \\
\hline & 6 & 3,2 & & \\
\hline & 7 & 3,5 & & \\
\hline \multirow{3}{*}{ Resolución de problemas } & 8 & 3,4 & \multirow[t]{3}{*}{3,4} & \multirow[t]{3}{*}{ Medio } \\
\hline & 9 & 3,4 & & \\
\hline & 10 & 3,6 & & \\
\hline \multirow[t]{3}{*}{ Sistema } & 11 & 3,5 & \multirow[t]{3}{*}{3,5} & \multirow[t]{3}{*}{ Medio } \\
\hline & 12 & 3,3 & & \\
\hline & 13 & 3,7 & & \\
\hline \multirow[t]{3}{*}{ Actitud ante el cambio } & 14 & 3,6 & \multirow[t]{3}{*}{3,6} & \multirow[t]{3}{*}{ Medio } \\
\hline & 15 & 3,6 & & \\
\hline & 16 & 3,5 & & \\
\hline \multirow[t]{3}{*}{ Capacitación } & 17 & 3,9 & \multirow[t]{3}{*}{3,6} & \multirow{3}{*}{ Medio } \\
\hline & 18 & 3,5 & & \\
\hline & 19 & 3,5 & & \\
\hline \multirow[t]{3}{*}{ Uso de la comunicación } & 20 & 3,7 & \multirow[t]{3}{*}{3,5} & \multirow[t]{3}{*}{ Medio } \\
\hline & 21 & 3,2 & & \\
\hline & 22 & 3,2 & & \\
\hline \multirow{2}{*}{ Interacción con el entorno } & 23 & 3,1 & \multirow{2}{*}{2,9} & \multirow{2}{*}{ Medio } \\
\hline & 24 & 2,5 & & \\
\hline
\end{tabular}

Fuente: Elaboración propia con la información recolectada, 2016. 


\section{Discusión}

Los docentes encuestados apuestan por el pensamiento sistémico de la quinta disciplina (entendida como una senda de desarrollo para la adquisición de actitudes y competencias), por ser la integradora de las demás doctrinas o postulados (los modelos mentales, el aprendizaje en equipo y el dominio personal para realizar su potencial), sin embargo, los docentes creen medianamente importante la práctica de la visión compartida (ligada al carisma del líder y a su habilidad para infundir una visión clara de futuro y al verdadero compromiso de todos), es decir, la creencia genuina de un futuro común, para el logro de los objetivos de las instituciones educativas, en otras palabras poco alientan el compromiso con las metas grupales, las que han mostrado culminar en resultados extraordinarios (Goodman, 1988; Senge, 2005; Becerra y Sánchez, 2011; Gómez, 2012).

Esto puede atribuirse, a que las instituciones educativas venezolanas generalmente adoptan visiones impuestas desde niveles jerárquicos educativos superiores, a pesar que los modelos de organizaciones exitosas son construidos sobre la base de las visiones de los integrantes de la organización, de allí la necesidad de estructuras educativas que dentro de su plan educativo nacional incluyan la flexibilidad del accionar, entre ellos los liceos bolivarianos venezolanos (diseñan y ejecutan proyectos formativos en acuerdo con problemas de la comunidad, sin abandonar la enseñanza de los aspectos técnicos formales), donde sus miembros son quienes determinan los objetivos a lograr en perfecto acuerdo con la realidad del entorno social donde se encuentra la institución educativa (Aguerrondo, 1992; Bolívar, 2000; Ministerio de Educación y Deportes, 2004; Ministerio del Poder Popular para la Educación, 2007).

También consideran el aprendizaje en equipo relevante, aunque medianamente para la conquista de las metas educativas, como figura más amplia que transciende la representación individual hacia el desarrollo de actitudes de grupos de individuos. Las facetas individuales, o que involucran necesariamente al ser, para el grupo estudiado, tienen mayor relevancia que las grupales, excepto el pensamiento sistémico, en este sentido hacen hincapié en el tratamiento de los modelos mentales para develar las limitaciones de la manera actual de ver el mundo, como paso previo para aceptar cambios en caso de que fuese necesario (una vez superados los supuestos arraigados en cada individuo, que le impedían aceptar otros puntos de vista, ampliar y diversificar su propia visión del mundo), con base en el dominio personal que impulsa la motivación para el continuo aprendizaje de la forma en que los actos propios afectan el entorno al que se pertenece (Senge, 2005). 
Al respecto, se indica que el dominio personal constituye el cimiento espiritual de la organización; debido a la importancia de lo emocional y racional en la interacción entre seres humanos para lograr un ambiente armónico que permita direccionar las energías hacia la consecución de los objetivos institucionales planteados, con base en la satisfacción, recepción del aprendizaje, conexión del propio aprendizaje con el del colectivo y aporte del conocimiento útil, hacia una organización educativa donde sus miembros experimenten el deseo de aprender y mejorar, superior en muchos casos al deseo de su propia institución, pero que no vaya en su contra (Senge, Kleiner, Roberts, Ross y Smith, 1995; Senge, 2005; Casell y Perea, 2009).

Se hace necesario destacar que aún cuando el dominio personal constituye una disciplina del ser dependiente del ejercicio individual, requiere también del apoyo de la organización, pero pocas organizaciones aprovechan el talento humano de sus integrantes, por tanto, se sienten poco valorados e incentivados por su institución, entonces eclipsa su espíritu de superación y con él su compromiso con la misma (Senge, 2005; Casell y Perea, 2009). Asimismo, puede señalarse, como se reporta en otras investigaciones que, en términos generales los docentes encuestados concuerdan con los postulados de la quinta disciplina como herramienta organizacional para afrontar los cambios evidenciados en los paradigmas educativos (Cardozo, 2003; Senge, 2005; Lugo, 2007; Mesa, 2008).

Los docentes encuestados consideran medianamente útil en la formación de ciudadanos de alto nivel técnico y humano, el asumir las características de las organizaciones que aprenden, es decir, del liceo como organización inteligente, para ellos es suficiente con que la organización a la que pertenecen sobreviva, no es imperativo que la institución educativa se enfoque en el aprendizaje generativo (el que caracteriza a la organización inteligente), es suficiente con el aprendizaje para la supervivencia, no creen fehacientemente en la organización inteligente como el ámbito donde la gente descubre continuamente cómo crea su realidad y como puede modificarla para expandir su capacidad para determinar su futuro, resta importancia, o no atribuyen la que realmente corresponde a la capacidad creativa que los individuos agrupados y motivados tienen (Llano, 1998; Gil, 2006; Martínez, 2006; Covey, 2009; Schatten, Seva y Tomicic, 2016).

Esto a pesar que la organización inteligente, como resaltan sus postulados, revela el camino para motivar a sus miembros a compartir su conocimiento y a la vez ser receptivos al saber-hacer de los otros que conforman el grupo, en procura del entorno constructivista del conocimiento colectivo, donde las personas, como elementos valiosos, edifican sus propias 
redes de inteligencia adaptativa para generar un patrón de conductas favorables en el diagnóstico de fallas organizacionales y de sus posibles soluciones, a través, de la práctica de amplia información para todos sus integrantes, libertad de acción y gobierno corporativo. En pocas palabras los integrantes del plantel deben ser libres, dueños de sus herramientas; así como de sus creaciones, pues los equipos competitivos así lo hacen, en plena ratificación del pensamiento sistémico, como enfoque estratégico a mediano y largo plazo para lograr verdaderas soluciones (Pinchot y Pinchot, 1999; León, et al.2003; Lazzarini, Albano, Arriaga, Lahitte y Lo Presti, 2004; Gil, 2006; Herrán, 2010).

El nivel medio de importancia que los docentes estudiados otorgan a la comunicación no comulga completamente con los postulados que al respecto se esbozan en la organización inteligente y en la mayoría de las estrategias gerenciales hasta ahora descritas, porque ella es indispensable para lograr la mayor eficiencia para el trabajo en equipo, asegurando el mejor desempeño de cada sujeto en las diferentes áreas, toda vez que la comunicación oportuna, directa y continua supone la interpretación adecuada de la información, mejor actitud ante el cambio, adecuado uso del conocimiento e interacción con el entorno, en procura de soluciones a problemas sociales, particularmente desde la esfera educativa. Así pues, la creación de flujos de información y conocimiento a partir de la política de mente abierta, permitirá el mejoramiento de la capacidad de respuesta del equipo a los problemas y necesidades planteados dentro y fuera de la organización (Ayón, 2006; Gil, 2006; Covey, 2009; Smits y Bowden, 2015).

Se concluye que las disciplinas individuales de la quinta disciplina, dominio personal y modelos mentales; y la colectiva, el pensamiento sistémico, son apreciadas por los docentes de alto valor como herramientas gerenciales en el desarrollo o consecución de la organización educativa inteligente, a pesar de que esta última tienen un nivel medio en la formación de adolescentes en instituciones educativas centradas en el diseño e implementación de proyectos que persiguen dar solución a problemas comunitarios. Por lo hallado se recomienda ampliar el número de indicadores a considerar en futuras investigaciones, para recomendar su implementación en el sistema educativo venezolano, porque las organizaciones educativas en la actualidad requieren de nuevas perspectivas para afrontar los desafíos que continuamente surgen en materia de formación. 


\section{Referencias}

Aguerrondo, Inés. (1996). La escuela como organización inteligente. Buenos Aires, Argentina: Editorial Troquel Educación.

Aguerrondo, Inés. (1992). La escuela media transformada: Una organización inteligente y una gestión educativa. Buenos Aires, Argentina: Ministerio de Cultura y Educación de la Nación, Dirección Nacional de Gestión de Programas y Proyectos.

Ayón, Rosa. (2006). Importancia de la comunicación en las organizaciones, un sistema de comunicación eficiente y un experto en comunicación que lo administre. Recuperado de https://es.calameo.com/read/00432433599b1b1951913

Bastidas, Gilberto, y Pacheco, José. (2011). Gerencia participativa y la promoción social en escuelas urbanas, municipio Trujillo, estado Trujillo, Venezuela. Revista Educación, 35(2), 1-30.

Becerra, Marlene, y Sánchez, Ludy. (2011). El liderazgo en las organizaciones inteligentes. Revista Científica Digital del Centro de Investigación y Estudios Gerenciales, 1(4), 61 71.

Bolívar, Antonio. (2000). Los centros educativos como organizaciones que aprenden. Promesa y realidades. Madrid, España: La Muralla.

Carballo, Juan, Masís, Mario, Sárraga, Gerardo, y Solís, Victor. (2008) ¿Pueden las organizaciones burocráticas ser organizaciones inteligentes y estar basadas en procesos y proyectos? San José, Costa Rica: Instituto Centroamericano de Administración Pública.

Cardozo, José. (2003). Propuesta de capacitación y desarrollo del personal administrativo basado en el enfoque gerencial de la quinta disciplina (Tesis de Maestría). Universidad Nacional Experimental Rafael María Baralt, Trujillo, Venezuela.

Casell, Ela, y Perea, Evelyn. (2009). Organizaciones Necias o Inteligentes. La Habana, Cuba: Universidad de La Habana.

Covey, Stephen. (2009). Los siete hábitos de la gente altamente efectiva. Barcelona, España: Paidós Editorial.

Furguerle, Johel, Pacheco, José, Hernández, Alba y Bastidas, Gilberto. (2016). Estrategias gerenciales en la educación básica y participación de madres y padres. Educare, 20(2), 1-20. Recuperado de http://www.redalyc.org/html/1941/194144435001/

Gil, Jaime. (2006). La gestión empresarial bajo el enfoque de las organizaciones inteligentes en la sociedad de la información. Negotium, 2(6), 33-54.

Gómez, Angel. (2012). Statistical-methodological proposal to measure organizational intelligence, based on the fifth discipline by Peter Senge. Negotium, 9(22), 53-83. 
Goodman, Michael. (1988). Study notes in system dynamics. Cambridge, Estados Unidos: MIT Press.

Guizar, Rafael. (2004). Desarrollo organizacional. México: Editorial Mc Graw Hill Interamericana.

Herrán, Agustín. (2010). Hacia las organizaciones que maduran: Teoría de los sistemas evolucionados aplicada a la evolución creativa de las organizaciones docentes. Madrid, España: Editorial Tendencias Pedagógicas.

Lazzarini, Héctor, Albano, Sergio, Arriaga, María, Lahitte, Mariana, y Lo Presti, Mónica. (2004). Organización en Red: Una forma inteligente de crecer. Novenas Jornadas "Investigaciones en la Facultad" de Ciencias Económicas y Estadística. Universidad de Rosario. Madrid, España: Editorial Electrónica Lazzarini Learning Systems.

León, Roger, Tejada Ebert y Yataco Marco. (2003). Las organizaciones inteligentes. Notas Científicas, 6(2), 82-87.

Llano, Alejandro. (1988). Organizaciones inteligentes en la sociedad del conocimiento. Cuadernos, empresas y humanismo. Espacios, 61(21). Recuperado de http://www.revistaespacios.com/a00v21n02/43002102.html

Lugo, Delia. (2007). Ambiente organizacional y resistencia al cambio en los directivos y docentes de la educación básica (Tesis de Maestría). Universidad Nacional Experimental Rafael María Baralt, Trujillo, Venezuela.

Martínez, Noemí. (2006). Gestión del conocimiento. Aprendizaje individual versus aprendizaje organizativo. Intangible Capital, 13(2), 308-326.

Mesa, Gabriel. (2008). La quinta disciplina como estrategia gerencial en la aplicación del proyecto educativo integral comunitario (Tesis de Maestría). Universidad Nacional Experimental Rafael María Baralt, Trujillo, Venezuela.

Ministerio de Educación y Deportes. (2004). Liceo Bolivariano, adolescencia y juventud para el desarrollo endógeno y soberano. Caracas, Venezuela. Recuperado de http://www.oei.es/quipu/venezuela/Liceo Bolivariano.pdf

Ministerio del Poder Popular para la Educación. (2007). Propuesta: Diseño curricular del sistema educativo bolivariano. Caracas, Venezuela: Edición Fundación CENAMEC. Recuperado de http://www.oei.es/quipu/venezuela/dl 908 69.pdf

Pinchot, Gifford, y Pinchot, Elizabeth. (1999). The intelligent organization: Engaging the talent \& initiative of everyone in the workplace. San Francisco, Estados Unidos: Editorial Berrett-Koehler

Schatten, Markus, Seva, Jurica, y Tomicic, Igor. (2016). A roadmap for scalable agent organizations in the Internet of Everything. Journal of Systems and Software, 115, 3141. 
Senge, Peter. (2005). La quinta disciplina del arte y la práctica en la organización. New York, Estados Unidos: Editorial Kairos S.A.

Senge, Peter, Roberts, Charlotte, Ross, Richard, Smith, Bryan, y Kleiner, Art. (1995). La quinta disciplina en la práctica. Estrategias y herramientas para construir la organización abierta al aprendizaje. Barcelona, España: Granica.

Senge, Peter. (1993). La quinta disciplina. Buenos Aires, Argentina: Granica Editorial.

Senge, Peter. (1990). La quinta disciplina: el arte y la práctica de la organización abierta al aprendizaje. Buenos Aires, Argentina: Granica/Vergara.

Smits, Stanley y Bowden, Dawn. (2015). A Perspective on Leading and Managing Organizational Change. Economics and Business Review, 1(15), 3-21. 